\title{
neurotic: Neuroscience Tool for Interactive Characterization
}

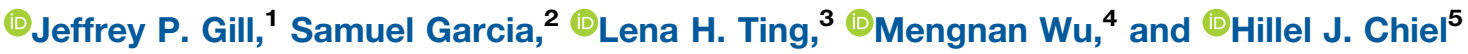

\author{
https://doi.org/10.1523/ENEURO.0085-20.2020
}

${ }^{1}$ Department of Biology, Case Western Reserve University, Cleveland, $\mathrm{OH} 44106-7080,{ }^{2}$ Centre de Recherche en Neuroscience de Lyon, Centre National de la Recherche Scientifique Unité Mixte de Recherche 5292- Institut National de la Santé et de la Recherche Médicale U1028-Université Claude Bernard Lyon 1, Lyon, 69675 Bron CEDEX, France, ${ }^{3}$ The Wallace H. Coulter Department of Biomedical Engineering, Emory University and Georgia Tech, Department of Rehabilitation Medicine, Division of Physical Therapy, Emory University School of Medicine, Atlanta, GA 30322-1119, ${ }^{4}$ The Wallace H. Coulter Department of Biomedical Engineering, Emory University and Georgia Tech, Atlanta, GA 30322-1119, and ${ }^{5}$ Departments of Biology, Neurosciences, and Biomedical Engineering, Case Western Reserve University, Cleveland, $\mathrm{OH} 44106-7080$

\begin{abstract}
A software tool for synchronization of video with signals would be of broad general use to behavioral neuroscientists. A new program, called neurotic (NEUROscience Tool for Interactive Characterization), allows users to review and annotate signal data synchronized with video, performs simple initial analyses including signal filtering and spike detection, is easy to use, and supports a variety of file formats. The program also facilitates collaborations by using a portable specification for loading and processing data and retrieving data files from online sources. Two examples are shown in which the software is used to explore experimental datasets with extracellular nerve or muscle recordings and simultaneous video of behavior. The configuration specification for controlling how data are located, loaded, processed, and plotted is also summarized. Algorithms for spike detection and burst detection are demonstrated. This new program could be used in many applications in which behavior and signals need to be analyzed together.
\end{abstract}

Key words: behavioral neuroscience; data sharing; open source; Python; video synchronization; visualization

\section{Significance Statement}

Behavioral neuroscience would benefit from a tool that allows easy visualization of both behavior and neural or other signals. A new tool, called neurotic (NEUROscience Tool for Interactive Characterization), is described that is free and open-source and can make data visualization, analysis, and collaboration much easier.

\section{Introduction}

In behavioral neuroscience, to interpret data after it is collected, it is essential that behavioral video be synchronized with electrophysiology. Although some commercial data acquisition systems may provide this functionality using proprietary hardware, software, and data formats [e.g., Plexon CinePlex Software (Jacobson et al., 2014), Blackrock Microsystems NeuroMotive], and although specialized tools exist for separately analyzing signals or behavioral videos

Received March 6, 2020; accepted April 16, 2020; First published April 24, 2020.

The authors declare no competing financial interests. using licensed analysis programs like MATLAB (http://www. mathworks.com/solutions/neuroscience), there are few free and open-source software tools with the capability to synchronize video and neural signals. Although there are many open source neuroscience tools and libraries for analysis of neural signals alone [e.g., Open Ephys (http://open-ephys. org) and NeuralEnsemble (http://neuralensemble.org) are communities focused on developing open source tools for

Author contributions: J.P.G., H.J.C., and L.H.T. designed research; J.P.G. and L.H.T. performed research; J.P.G. and S.G. contributed unpublished reagents/analytic tools; J.P.G., H.J.C., L.H.T. and M.W. analyzed data; J.P.G., H.J.C., L.H.T. and M.W. wrote the paper. 
neuroscientists], they often have steep learning curves and require that users program their own scripts. Especially appealing would be an open source application that facilitated the review of electrophysiology data with synchronized video, was simple to install on any computer, easy for nonexperts to use, and powerful enough for experienced data scientists to use in their data pipelines.

We have developed neurotic (NEUROscience Tool for Interactive Characterization), a Python tool with these capabilities (for important links, see Table 1). A graphical user interface (GUI) provides interactive plots of raw electrophysiology data, simultaneous video, and simple annotation tools. The program also performs basic data processing, like filtering and spike detection. Since it is built on the Neo library (Garcia et al., 2014), it can read many common electrophysiology file formats. The application is easily installed using common Python tools (pip or conda) or standalone installers, with built-in examples running quickly. After creating human-readable configuration files, users can explore data with the GUI. Portable configuration files and a data-fetching feature facilitate collaboration. Programmers can bypass the GUI and use the application programming interface (API) in their analysis pipelines in scripts and Jupyter notebooks (Kluyver et al., 2016).

\section{Materials and Methods}

\section{Software overview}

neurotic relies on the Python library Neo (Garcia et al., 2014) for reading electrophysiology files, including formats like Axon, AxoGraph, Blackrock, Intan, and Open Ephys files; it also includes some generic formats like MATLAB MAT-files, HDF5, plain text ASCII, and generic binary data files, provided they are structured properly. Neo manages units (e.g., ensuring signals measured in millivolts and microvolts are summed correctly) using the quantities package (http://pypi.org/project/quantities), and both rely on the ubiquitous Numpy library (van der Walt et al., 2011). neurotic can take advantage of a feature of many of Neo's file readers, which is to read slices of large files as needed, rather than loading entire files into memory at once; this "fast loading" feature greatly reduces load times and memory consumption.

neurotic reads spreadsheet files (CSV format) using the Python library pandas (McKinney, 2010) to import epochs and events. Epochs are labeled periods of time with a

This work was supported by the National Science Foundation Integrative and Organismal Systems Israel Science Foundation (NSF-IOS-BSF) Grant 1754869 and U.S.-Israel Binational Science Foundation Grant 2017624.

Acknowledgements: We thank collaborators who have provided feedback on early versions of the application. Beam walking data was collected in the Neuromechanics lab at Georgia Tech and Emory University by Luke Drnach, Sistania Bong, Mengnan Wu, and Lena Ting.

Correspondence should be addressed to Jeffrey P. Gill at jeffrey.p.gill@ gmail.com.

https://doi.org/10.1523/ENEURO.0085-20.2020

Copyright @ 2020 Gill et al.

This is an open-access article distributed under the terms of the Creative Commons Attribution 4.0 International license, which permits unrestricted use, distribution and reproduction in any medium provided that the original work is properly attributed.
Table 1: Important links

\begin{tabular}{ll}
\hline \multicolumn{1}{c}{ Resource } & \multicolumn{1}{c}{ URL } \\
Source code & https://github.com/jpgill86/neurotic \\
Documentation & https://neurotic.readthedocs.io \\
PyPI distribution & https://pypi.org/project/neurotic \\
Anaconda & https://anaconda.org/conda-forge/ \\
distribution & neurotic \\
$\begin{array}{c}\text { Standalone } \\
\text { installers }\end{array}$ & $\begin{array}{c}\text { https://github.com/jpgill86/neurotic/ } \\
\text { releases }\end{array}$ \\
Issue tracker & https://github.com/jpgill86/neurotic/issues \\
\hline
\end{tabular}

beginning and an end which are used to annotate data by labeling periods of interest. Events are labeled points in time that serve as "bookmarks" into the experiment, allowing users to jump to them. The neurotic interface allows users to create and save epochs to a spreadsheet.

neurotic can read many video file formats using the PyAV library (http://pypi.org/project/av). Sound stored in video files is currently ignored by neurotic.

neurotic can perform processing and analysis procedures on the data, including applying filters, generating spike trains from signal peaks, and identifying bursts (periods of high activity in the spike trains). These procedures are aided by the Python library elephant (http://pypi.org/ project/elephant), which provides advanced algorithms for analyzing Neo objects. Many of these algorithms are applications of signal processing procedures implemented in the SciPy package (Virtanen et al., 2020).

Finally, neurotic uses the Python library ephyviewer (http://pypi.org/project/ephyviewer) for plotting and interacting with signals, epochs, events, spike trains, and video frames. ephyviewer is built on the pyqtgraph library (http://www.pyqtgraph.org), which creates a Qt application interface (www.qt.io). Figure $1 A$ shows the neurotic user interface for an example dataset in which neural and force data were collected as an animal fed (Gill and Chiel, 2020). Electrophysiology signals are plotted after filtering, and points mark peaks identified by window discriminators. Video shows the animal's behavior, and the timing of the displayed frame is indicated by a vertical line superimposed on the plots. A navigation panel allows the user to simultaneously move through the neural data and synchronized video. Another panel ("epoch encoder") displays user-created epochs as horizontal bars and provides controls for creating and modifying epochs (collapsed in Fig. 1A; for details, see Fig. 1D). A list of user-designated events makes it possible to jump to moments of interest.

\section{Software configuration}

neurotic requires configuration before use with a dataset. For example, the paths to the electrophysiology data file and behavioral video file must be specified. Configurations are saved in the human-readable YAML file format which can be used to load and process data repeatedly.

Figure 2 shows an example of a detailed configuration written in the YAML format. A minimal configuration could be much simpler, including, for instance, a single file path. 
A

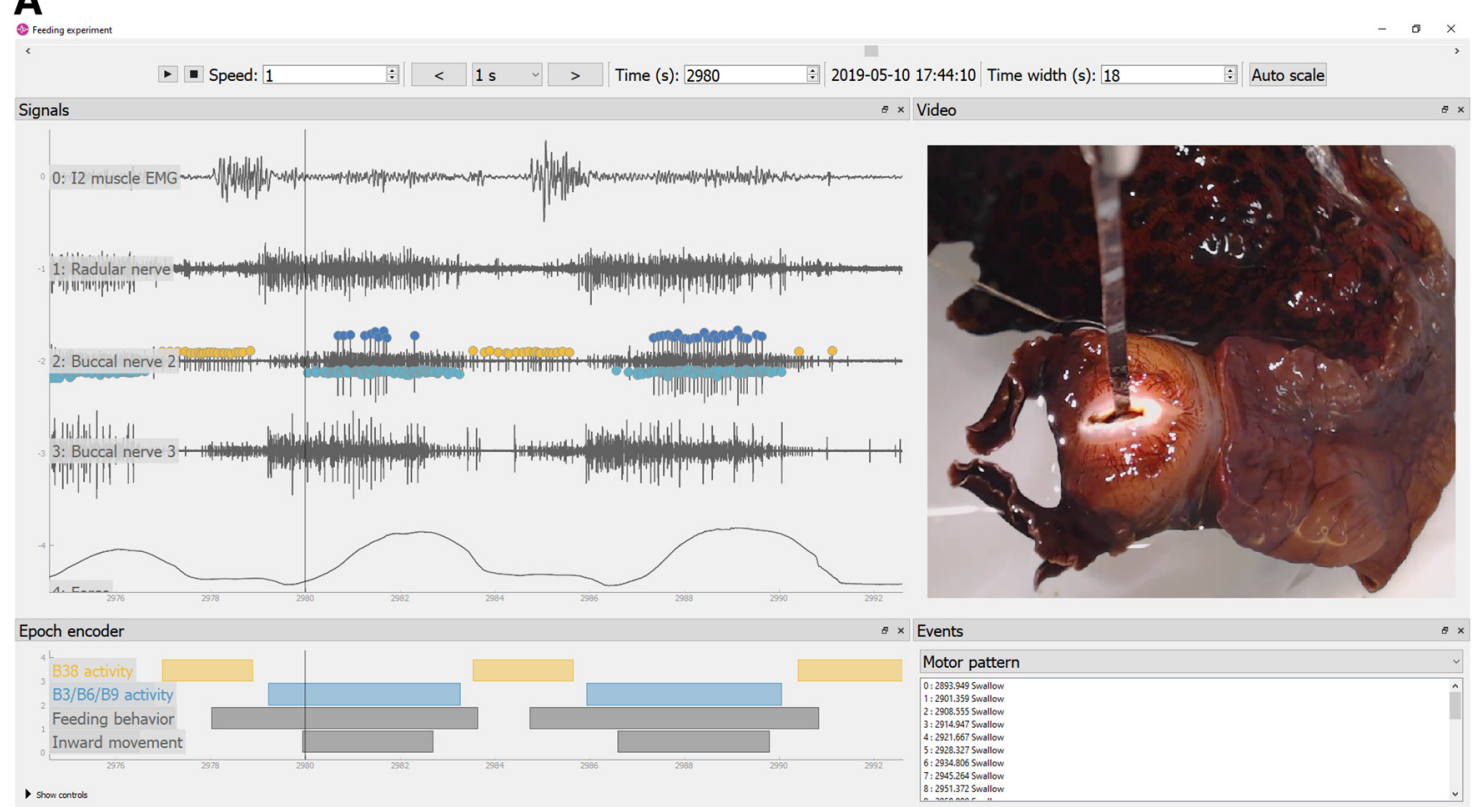

B

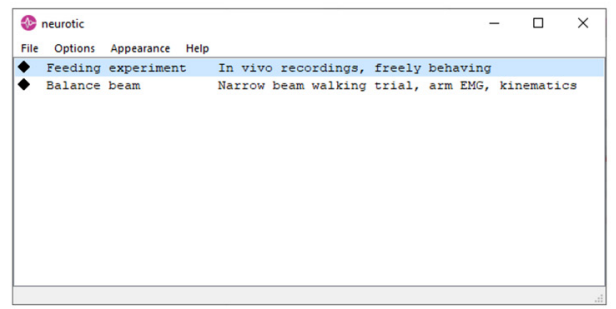

\section{C}

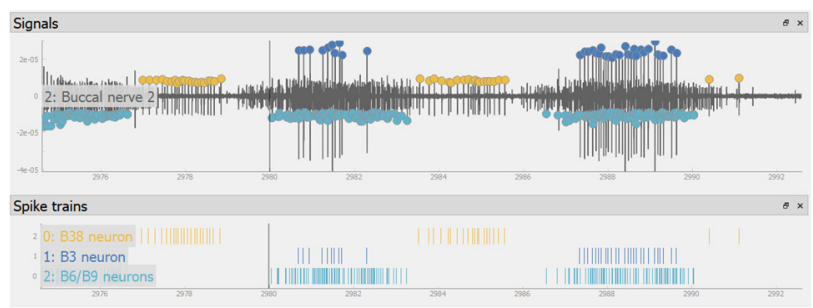

D

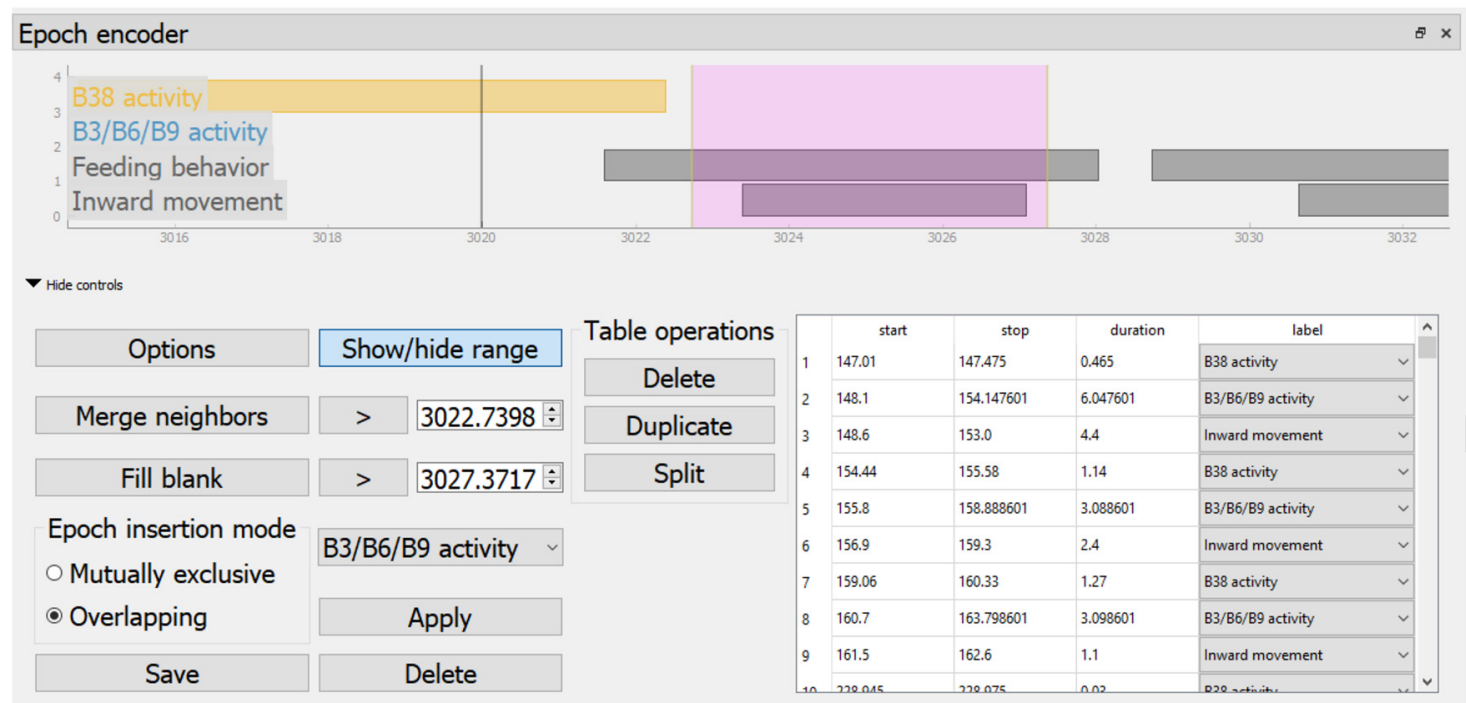

Figure 1. GUI. The GUI of neurotic combines data from multiple sources, such as electrophysiology data files and behavioral video files, into a unified and synchronized view for reviewing and annotating experimental data. This example shows data collected as an animal (Aplysia californica) ate seaweed (see Materials and Methods, Experimental methods). Implanted electrodes captured extracellular signals from a muscle and three nerves associated with feeding. Video of behavior and swallowing force from food attached to a force transducer were also recorded. $\boldsymbol{A}$, The interface has movable and resizable panels created using the Python package ephyviewer (http://pypi.org/project/ephyviewer). Across the top of the interface, a time navigation bar allows a user to jump through the record or play it at different speeds (keyboard shortcuts provide similar features). The "time width" box controls the displayed duration. Muscle, nerve, and force signals are displayed (top left panel) along with the video (top right panel). A vertical line indicates 
continued

the time corresponding to the displayed video frame. Panels can be zoomed using the mouse, and time can be expanded or contracted using the mouse or the time width input. User-defined events are listed (bottom right panel), providing bookmarks to different parts of the experiment. A tool called the epoch encoder (bottom left panel) allows users to label time periods (controls are collapsed here; see $\boldsymbol{D}$ for details). Some panels can be double-clicked to open dialogue windows with additional options (e.g., to hide plotted signals). $\boldsymbol{B}$, Before displaying a user interface like that in $\boldsymbol{A}$, neurotic provides a window for selecting datasets. Users load a dataset list from a manually created human-readable YAML configuration file that provides paths to data files and other parameters (Fig. 2). If data and video files are available on the internet, neurotic can be configured to locate and download files if they are not already available locally. Icons to the left of each dataset name indicate whether files are already available locally or not. This window provides menus for loading options and color themes. The most important of these is the toggleable fast loading option, which is available for a subset of data file types and provides much faster loading with less memory consumption in exchange for skipping data processing steps like filtering and spike detection. $\boldsymbol{C}$, Using optional parameters provided in the configuration file (Fig. 2G), peaks can be detected on signals using amplitude-threshold window discriminators (see Fig. 3 for algorithm). Peaks identified by the same discriminator are grouped as a unit and plotted both as points on the signals and as raster plots in a separate panel (hidden in $\boldsymbol{A}$ but shown in this panel). $\boldsymbol{D}$, The epoch encoder panel displays horizontal bars to represent user-created, labeled time periods. Periods of interest, such as a particular behavior, the phase of a rhythmic behavior, or the activity of an identified neuron may be labeled using the epoch encoder. These data are saved to a plain text spreadsheet format (CSV) that may be used for further analysis. The epoch encoder panel allows precise control of the temporal parameters of epochs, merging of adjacent epochs, and filling gaps between epochs. Epochs can be placed using the range selection feature (magenta rectangle) with the mouse by dragging its edges. Keyboard shortcuts exist for rapid encoding. A table lists the existing epochs and allows them to be modified or removed.

The example is divided into eight sections: (1) a unique dataset name and brief description; (2) file paths may be listed relative to a primary directory; (3) video synchronization parameters; (4) signal plotting parameters; (5) signal filtering; and settings for (6) the epoch encoder, (7) peak detection, and (8) burst detection.

Multiple configurations like that shown in Figure 2 may be contained in a single YAML file. This allows multiple datasets, or alternate processing and display configurations for the same dataset, to be contained within one file. When neurotic is first opened and the configuration file is selected, a list of dataset configurations is displayed (Fig. $1 B$ ). The user can choose which configuration to load and can adjust options for how data are loaded and displayed.

For downloadable datasets, including those on private password-protected servers or public data repositories like GIN (http://gin.g-node.org), the configuration may include URLs for the files, and neurotic can download them. In combination with relative file paths, this makes configuration files fully portable, such that users may share them with collaborators and load datasets from other computers.

Synchronizing a video file and a signal data file captured using independent data acquisition systems may be complicated, since files may start at different times or the video may contain multiple trials. neurotic provides several options for synchronization, including options for offsetting the video start time, adjusting frame rate, and skipping sections.

\section{Algorithms for data processing}

A simple algorithm for detecting peaks is illustrated schematically in Figure 3 . Figure $3 A$ plots nerve activity measured extracellularly (see below, Experimental methods). Each spike is an action potential, and the spike size is determined by the axon size, its proximity to the electrode, and its complement of ion channels.

Prior work in Aplysia has shown that the amplitude and timing of distinct units can identify neurons (Morton and
Chiel, 1993; Hurwitz et al., 1996; Lu et al., 2013, 2015; McManus et al., 2014; Cullins et al., 2015). Figure $3 B$ shows amplitude thresholds and epochs that together define windows for peak detection. Each amplitude discriminator has lower and upper thresholds, and may be constrained to labeled epochs. Identified peaks (Fig. 3C) are plotted in the neurotic interface as points and separately as a spike train raster plot (Fig. 1C).

High-frequency activity (bursts), defined by initiation and termination frequency thresholds, can be identified after peak detection. The result of applying burst detectors (configured in Fig. $2 \mathrm{H}$ ) to the example spike trains is shown by boxes enclosing bursts (Fig. 3D). In the neurotic interface, the timing of detected bursts is plotted as epochs.

neurotic can also calculate the rectified area under the curve (RAUC). This procedure optionally removes a baseline (mean or median) from a signal, performs full-wave rectification (absolute value), and then integrates the signal in bins of fixed duration. The integration step introduces smoothing (dependent on the bin size). The resulting time series is plotted in the neurotic interface. This helps visualize overall signal activity [e.g., electromyogram (EMG), which is known as integrated EMG (iEMG)].

\section{API for additional analyses, customization, and prototyping}

neurotic functions as a standalone application and also as a library with classes and functions that may be used in scripts or Jupyter notebooks. With neurotic's API, users can read configuration files, load data, and apply processing steps as in the GUI. The resulting data objects are represented using Neo's object model (Garcia et al., 2014) and may then be used for additional analyses. For example, after identifying peaks, creating spike trains, and detecting bursts using a neurotic configuration stored in a YAML file or in memory as a Python dictionary, a user might statistically analyze burst durations, plot inter-spike interval histograms, create publication-quality figures 


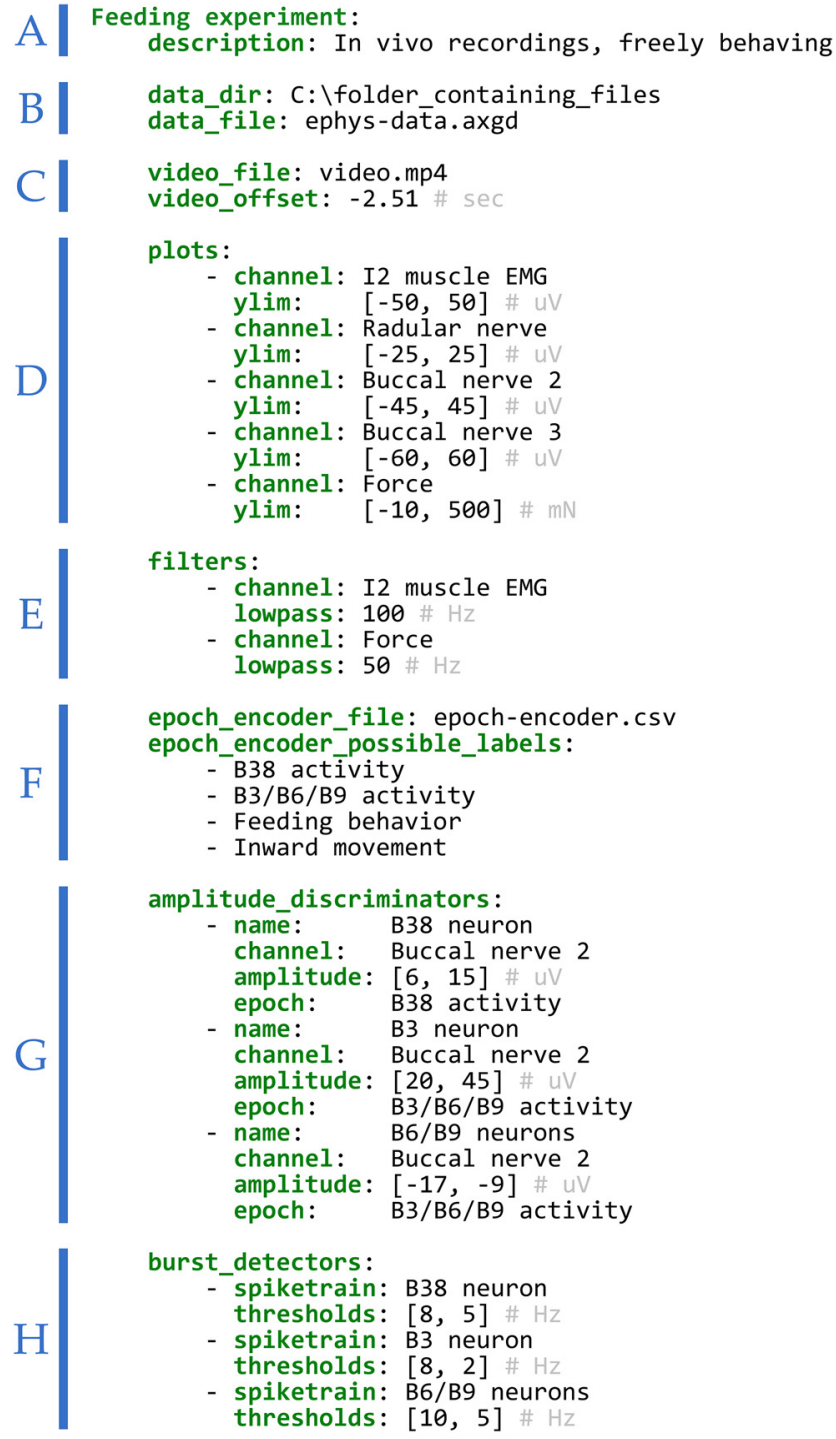

Figure 2. An example configuration file. To visualize experimental data with neurotic, datasets must be listed and described within human-readable plain text YAML configuration files. Multiple configurations for the same or different datasets may be listed within one file and are separated into blocks using blank lines and indentation. This example shows one configuration that is similar to that used to create Figures 1, 3. Each set of parameters shown is optional. $\boldsymbol{A}$, Each configuration is given a unique name, e.g., "feeding experiment." Details associated with the configuration are indented beneath the name. A brief description of the dataset may be provided. The names and descriptions of datasets are displayed when a YAML file is loaded, giving the user the option to select the dataset to view (Fig. 1B). $\boldsymbol{B}$, Paths to related files found locally on the computer, e.g., signals and video from a single experiment, may be located within a single directory specified by data dir (such a file tree structure is convenient but not necessary as neurotic can accept relative or absolute paths). A file containing signal data that is readable by the Python package Neo (Garcia et al., 2014) is specified using data file. Neo supports many file types (see list: https://neo.readthedocs.io/en/latest/io.html\#module-neo. io). Signals are read from the data file, processed according to other, optional configuration settings, and plotted. For example, in this figure, sections $\boldsymbol{D}, \boldsymbol{E}, \boldsymbol{G}$ affect plotting. $\boldsymbol{C}, \mathrm{A}$ video file using a plotting package, or export data as part of a larger analysis.

The neurotic package includes an example Jupyter notebook containing a tutorial which can serve as a starting point for new users, and documentation is provided for the API.

\section{Experimental Methods \\ Animal feeding experiments}

Briefly, the methods for obtaining neural correlates of responses to changing load during feeding in intact animals are described (for details, see Gill and Chiel, 2020).

Multichannel differential hook electrodes made from fine stainless steel wires $(25-\mu \mathrm{m}$ diameter, stainless steel 316, heavy polyimide insulated, California Fine Wire) were implanted in adult Aplysia californica (South Coast BioMarine; Cullins and Chiel, 2010). Animals were first anesthetized with an injection of isotonic (333 mm) magnesium chloride and immersed in an ice bath. An incision was made in the side of the head, and hook electrodes were attached using Kwik-Sil biocompatible silicone adhesive (World Precision Instruments) to nerves and muscles of the animal's feeding apparatus: the 12 protractor muscle, the radular nerve (RN), and buccal nerve 2 (BN2) and buccal nerve 3 (BN3). The incision was then sutured closed, and animals were allowed to recover for 1-3 $d$.

Animals were presented with food in the form of seaweed strips (Deluxe Sushi-Nori, nagai roasted seaweed, Nagai Nori USA INC). While animals fed, signals from the hook electrodes were amplified and filtered (Model 1700 Differential AC Amplifiers, A-M. Systems), digitized (NI PCle-6251 and NI BNC-2111, National Instruments), and recorded at $5000 \mathrm{~Hz}$ using AxoGraph X (AxoGraph Scientific) in continuous acquisition mode and saved in the AxoGraph file format, which is readable by Neo. In some trials, double-sided tape was placed between two seaweed strips to create an unbreakable strip, and this was attached to a force transducer (model FT03E, Grass

\section{continued}

may be associated with the signal data. Synchronization parameters can be given for controlling the video and signal data alignment. For example, in this case, video capture began 2.51 $s$ before signal data acquisition, so -2.51 is provided for video offset to shift the video start time. Parameters for correcting for frame rate inaccuracies or clipping the video are also available. $\boldsymbol{D}$, All signals or a subset of signals will be plotted according to the parameters given under plots, which control plot range and labeling (Fig. $1 A$, top left panel). $\boldsymbol{E}$, Signals may be filtered before plotting. $\boldsymbol{F}$, An optional epoch encoder GUI panel (Fig. 1D) creates annotations that may be saved to a spreadsheet (CSV) file. The epoch encoder allows user-defined labels to be attached to time periods. G, Peaks may be identified in the signals using amplitude thresholds. Each amplitude discriminator is applied to the specified signal channel and may be constrained to periods marked with a particular epoch label. This creates one spike train for each amplitude discriminator, plotted both as points on the signals and as a raster plot (Fig. 1C). See Figure 3 for algorithm details. $\boldsymbol{H}$, Bursts of activity in spike trains may be detected using initiation and termination firing frequencies. 

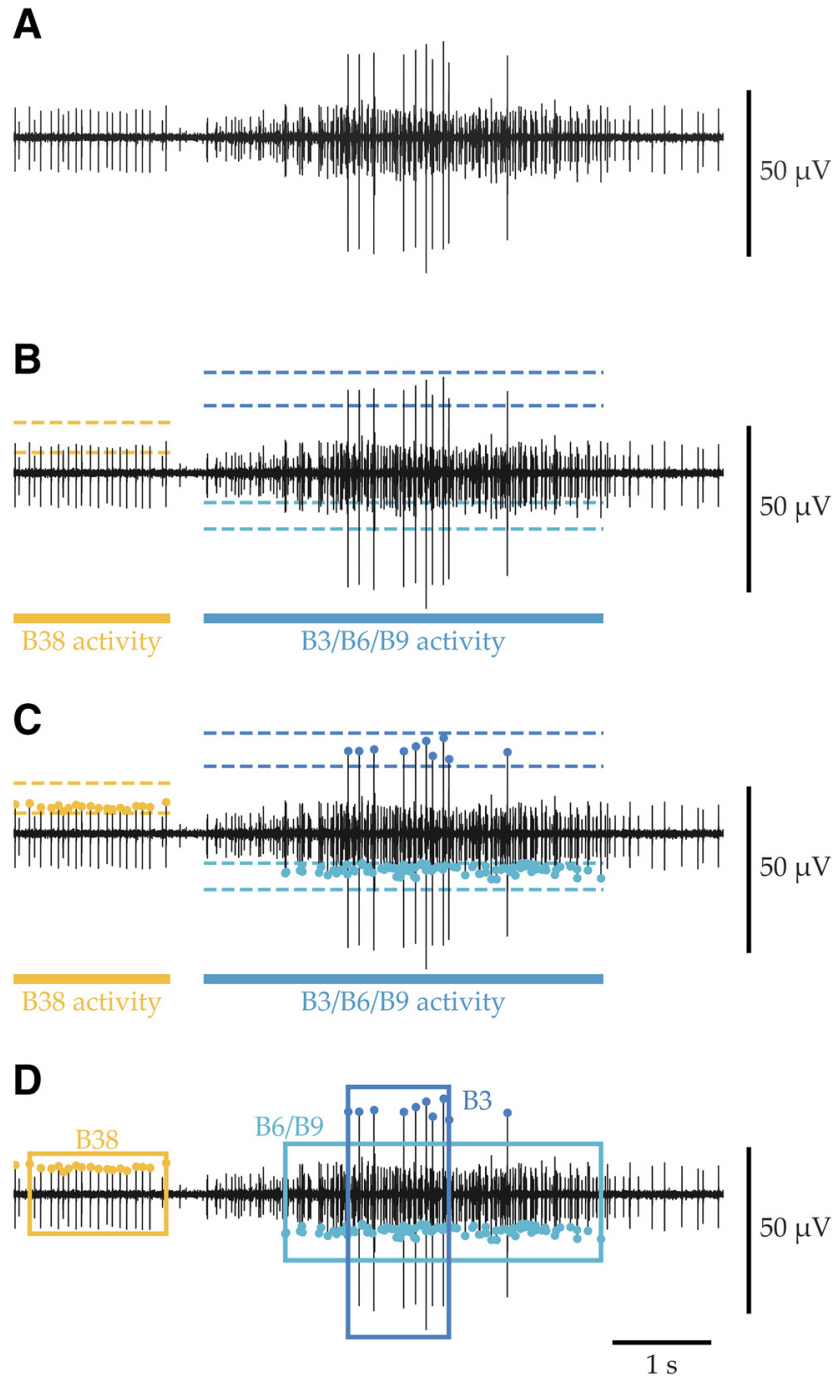

Figure 3. Peak detection using a window discriminator to define neuronal bursts. Panels show an example of applying three amplitude discriminators to a single channel to obtain three sets of peaks, classified as action potentials originating from identified motor neurons B38 (McManus et al., 2014), B6/B9, and B3 (Lu et al., 2015). The configuration settings are shown in Figure $2 \mathrm{G}$. $\boldsymbol{A}$, The raw signal obtained from an extracellular whole nerve recording of BN2 is plotted and contains activity from motor and sensory neurons. Identified neurons are distinguishable by their relative amplitude and timing within the feeding motor pattern (Lu et al., 2013). B, Epochs labeled "B38 activity" and "B3/B6/ B9 activity" were placed manually using the epoch encoder (Fig. 1D) based on prior work. Amplitude windows for spike classes were estimated manually and specified in the configuration file. $\boldsymbol{C}$, At load time, using algorithms from the Python package elephant (http://pypi.org/project/elephant), local maxima or minima were located within the given amplitude range and contained within a period with an appropriate epoch label. These points are displayed on the signals and as raster plots (Fig. 1C). $\boldsymbol{D}$, Subsets of each spike train were grouped into bursts defined by firing frequency thresholds (boxes). Notice that the initial B38 spike and the final B3 spike were excluded from the bursts because they did not meet the firing frequency conditions. Burst times may then be used for further analysis.
Instrument Division, Astro-Med Inc) oriented over the animal to measure downward pulling force during swallowing. Force was also amplified, low-pass filtered, digitized by a strain gauge conditioner (model 3170, Daytronic), and recorded using AxoGraph X.

Simultaneously, video of the behavior was captured at 30 frames per second using a webcam (Logitech HD Pro Webcam C920) and Logitech Webcam Software. Video was saved as MP4 files with H.264 video encoding. A digital counter (C342-0562 totalizing counter, Veeder-Root) was programmed to increment at $10 \mathrm{~Hz}$ when the recording software was started; the counter was visible in the video recording at the start and end of the experiment, and pulses to the counter were recorded in AxoGraph X. This allowed the difference in timing between the start of video acquisition and the subsequent start of signal acquisition to be determined after the experiment, ensuring that the video and signal data could be synchronized by neurotic.

\section{Human beam walking experiments}

The methods for assessing human balance performance and control strategies while walking on a narrow beam are described. The participant consented to the protocol approved by the Georgia Tech and Emory Institutional Review Boards.

The participant attempted to walk as far as possible across a narrow beam with left arm free and right arm held across the chest. The participant was instructed to stop if she uncrossed her right arm or stepped off the beam. No explicit instructions were given regarding stepping pattern or walking speed.

Video data, EMG signals from arm muscles, and kinematics of the body were recorded using a ten-camera motion capture system (Vicon). EMGs were obtained at $1200 \mathrm{~Hz}$ from surface electrodes placed over the following arm muscles: biceps, triceps, and wrist flexors and extensors. The 3-D locations of retro-reflective markers placed at the center of the clavicles and both heels were collected at $120 \mathrm{~Hz}$, along with time-synched video data (Vicon Bonita) collected at $40 \mathrm{~Hz}$ in $\mathrm{AVI}$ format and later converted to MP4 files.

\section{Results}

Using neurotic for reviewing behavioral video and synchronizing it with neural signals greatly reduces analysis time. Previously, alignment of video and neural signals, which were recorded and reviewed in separate programs with independent time bases, required that times of interest for every comparison had to be located separately in each program.

neurotic solves the data stream alignment problem for old and new datasets. Moving through the video and finding the corresponding signal is done automatically in a unified interface, allowing for continuous playback and skipping to different parts of the experiment, making review easier, faster, and less prone to error.

To illustrate the program's utility, we show alignment of behavior video with neural signals and force records (Fig. 4). Using neurotic, initial analysis of effects of mechanical 
A

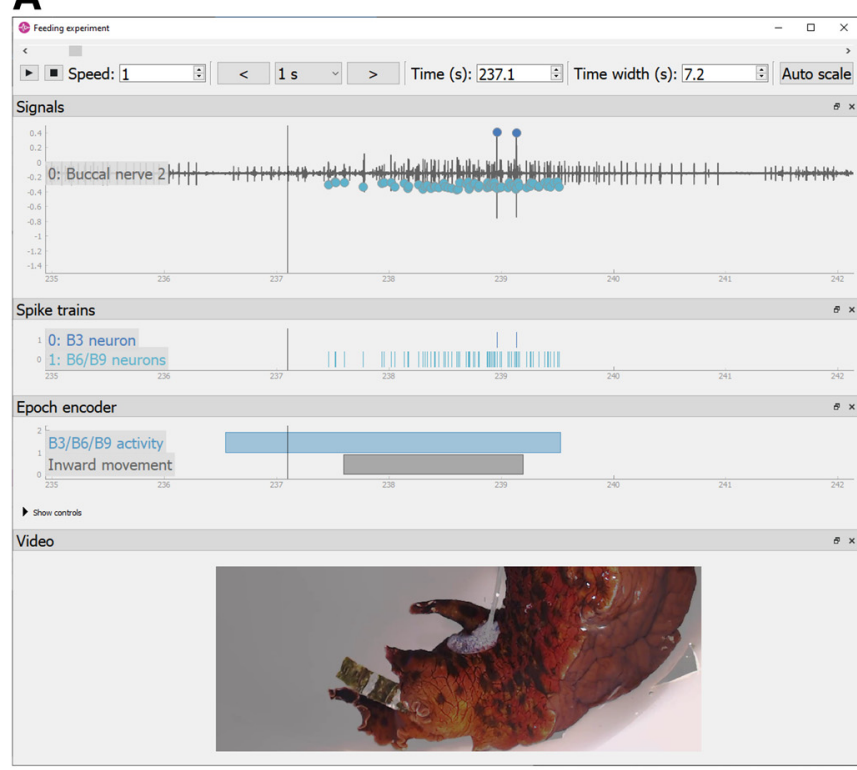

C

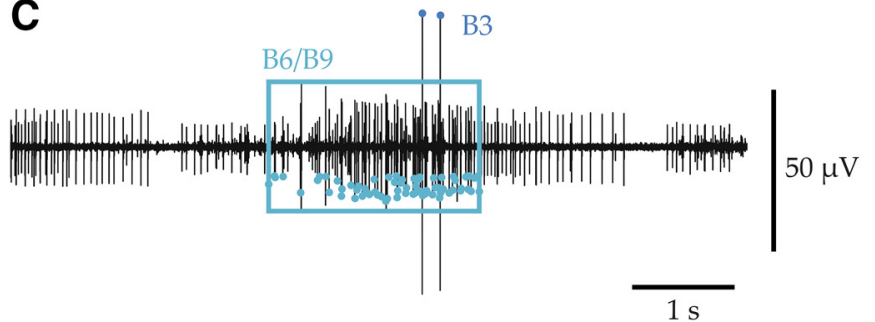

B

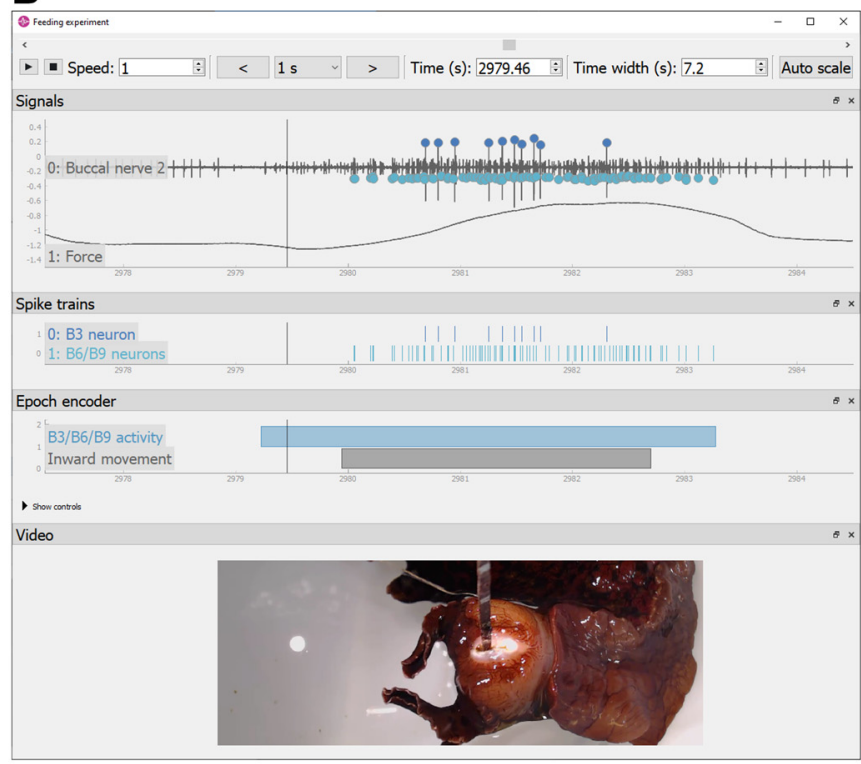

D

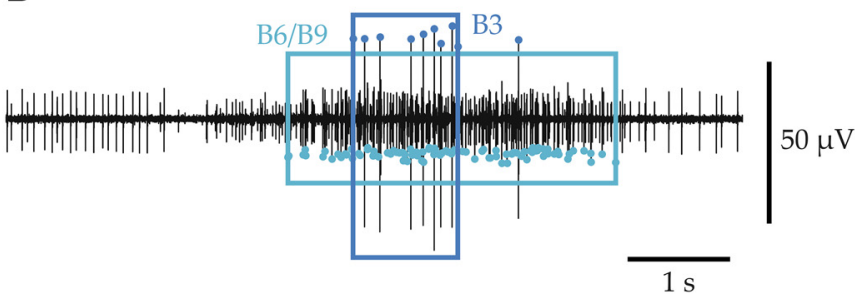

Figure 4. Using neurotic to explore an adaptive response to load during feeding. $\boldsymbol{A}$, As an animal swallowed a strip of unloaded (unanchored) seaweed, activity of identified feeding motor neurons B6/B9 and B3 was identified from the extracellular nerve recording of BN2. Using the synchronized video and epoch encoder, the timing of inward movement of the strip and the approximate period during which the identified neurons fired were labeled (gray and blue bars, respectively). Using the peak detection feature, mediumsized spikes were classified as B6/B9, and large spikes were classified as B3, which are major motor neurons known to contribute substantially to swallowing force (Lu et al., 2015). Spikes are plotted as points and in raster plots. $\boldsymbol{B}$, In a separate trial, the same animal fed on a strip of unbreakable seaweed anchored to a force transducer. The force generated by the animal is plotted with the extracellular nerve signals. In response to the increased load, the animal activated the identified motor neurons at high frequency for longer, leading to greater force. C, Using firing frequency criteria determined by prior work (Lu et al., 2015), all spikes generated when feeding on an unloaded seaweed strip associated with the B6/B9 motor neurons (light blue) were grouped into a burst. The two spikes generated by B3 (dark blue) did not meet the criterion. $\boldsymbol{D}$, Using the same criteria when the animal fed on a loaded strip, all B6/B9 spikes and all but one B3 spike were grouped into bursts. The data suggest that the B3 neuron is more likely to activate at high frequency when the animal encounters load, and the B6/B9 neurons are active for a longer duration (Gill and Chiel, 2020).

load on feeding in Aplysia was simplified. Swallow durations were greater in the presence of load, and motor pattern changes could be quickly surveyed during multiple responses. For example, one swallowing response under conditions of low load (Fig. 4A) or high load (Fig. 4B) could be easily compared; only one neural channel (BN2) is plotted for clarity, as is force of swallowing when the seaweed strip was attached to the force transducer (Fig. 4B). Using the synchronized video, the periods during which the animal pulled food into its mouth could be identified, and these periods could be labeled using the epoch encoder (gray bars). Using amplitude window discriminators, action potentials generated by identified motor neurons could be located and represented as spike trains (raster plots). Using user-specified firing rate thresholds, neurotic detected bursts in the spike trains (Figs. $4 C, D$ ), allowing the durations of bursts to be compared when load was low or high. In the example, the duration of intense B6/B9 motor neuronal activity is longer during swallowing on a high-load stimulus, and the B3 motor neuron is recruited.

neurotic has broad applications because it uses the Neo Python library for reading a wide variety of file formats. For example, Figure 5 shows human EMG and kinematic data, stored as a binary MATLAB MAT-file, as a person maintains her balance while walking across a narrow beam. Left arm muscle activation timing corresponds to the moments that the participant could be seen correcting for a momentary balance loss. Similarly, a decrease in right shoulder elevation is seen simultaneously in both the video and a digitized record of a body marker tracking shoulder position in 3-D space. 


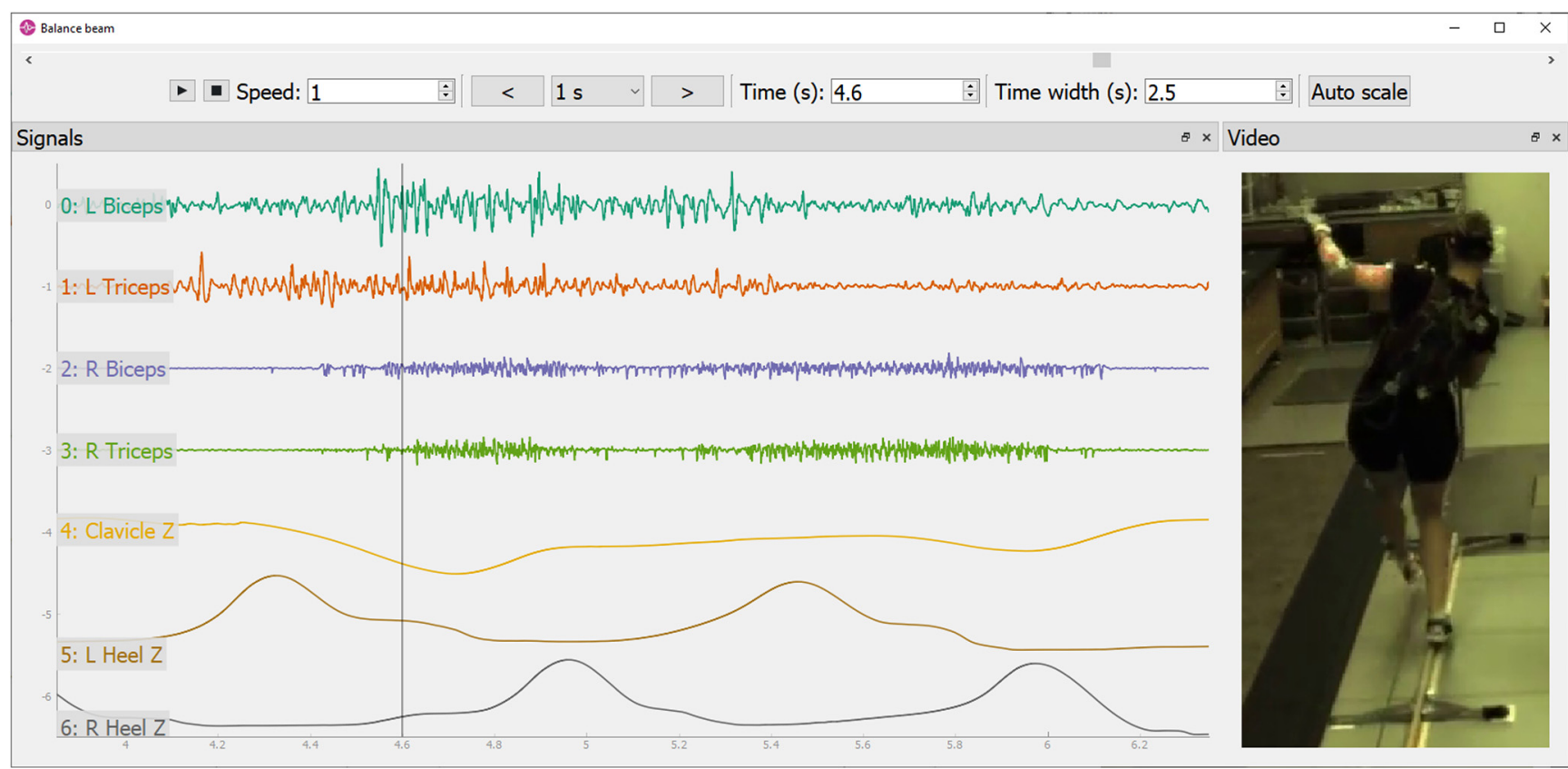

Figure 5. Using neurotic to explore balance beam walking EMG and kinematics. The vertical line corresponds to the time of the video frame shown where the participant lifts her left arm and extends it at the elbow as she regains balance while walking along a narrow beam (1.8 cm wide, $3.66 \mathrm{~m}$ long). Time-synched EMG signals show that the left triceps is activated before this event (second trace), consistent with extending the elbow, and then is followed by activation of the biceps (first trace), consistent with braking elbow extension. The $z$-coordinates (elevation) of the markers at the center of the clavicles and on each heel are also plotted. At the time of the illustrated video frame, the dip in clavicle elevation (fifth trace) is associated with the lowering of the participant's right side of the torso as part of the balancing response. The left heel marker (sixth trace) shows that the left leg was nearing the ground just before being placed on the beam. The right heel marker (seventh trace) shows a slow rise as the participant began to roll forward on the ball of her right foot.

\section{Discussion}

Using neurotic, behavioral and neural responses to stimuli can be identified and annotated rapidly and subjected to initial signal processing. Changes in neural activity during swallowing on unloaded and loaded food stimuli in Aplysia could be readily observed (Fig. 4), and relationships between human movement and EMG were deducible (Fig. 5). In addition, dynamical model data could be readily incorporated and compared with empirical physiological data.

Open source projects like this thrive on contributions from volunteer developers. Because neurotic uses the Neo package for reading electrophysiology files, members of the NeuralEnsemble community (http://neuralensemble. org) can readily contribute to its further development. In general, open source projects depend on an enthusiastic community of developers, and if neurotic attracts such a community, it will continue to develop. The source code is stored on GitHub, which provides valuable collaboration tools such as issue tracking, pull request management, conversations, and task delegation. The open source model allows anyone to suggest improvements, submit bug fixes, or contribute new features; the community of maintainers seeks consensus on whether to accept contributions or request alterations; finally, changes are accepted only after passing a review process and automated testing. The complete history of the code can be found on GitHub, and prior releases of the software will remain available on distribution platforms like PyPI and Anaconda
Cloud, so older versions of the software should always remain accessible.

There are trade-offs scientists should consider when choosing to use either free, open source software for their research or proprietary, closed source software. Commercial solutions that provide video synchronization capabilities similar to neurotic are few, and those proprietary alternatives may require expensive investments in software licenses and specific hardware. Maintaining proprietary systems depends on the continued financial viability of the company that makes them; old versions may stop working, and new hardware may be needed to upgrade software. For open source programs, maintainability again depends on the community. In some important ways, open source is superior for reproducibility and transparency, because the code is available for inspection, users suggest and often implement improvements to the code, and bugs are usually found and fixed quickly. Although proprietary systems often provide expensive "plugins" that can be added to the original software, open source projects excel at extensibility, because users can readily add functionality, or even specialize the program further for their own applications. The major investment for an open source project is the time that needs to be devoted to the project, whereas the major investment associated with proprietary software and hardware solutions is the money that needs to be invested into the hardware and software.

There are exciting possibilities for future development of neurotic. More signal processing options could be 
provided, such as customizable or even arbitrary multistage analysis procedures. Sound could be added to video playback. More advanced spike sorting techniques could be integrated [e.g., using results from tridesclous (tridesclous.readthedocs.io) or NeuroChaT (Islam et al., 2019)]. The pyqtgraph GUI library has many capabilities that could expand neurotic's interactive and annotation features. Performance could be improved by incorporating GPU acceleration using the VisPy library (http://www. vispy.org). Finally, thanks to the Neo community, the list of compatible file formats is continuously growing.

\section{References}

Cullins MJ, Chiel HJ (2010) Electrode fabrication and implantation in Aplysia californica for multi-channel neural and muscular recordings in intact, freely behaving animals. J Vis Exp. Advance online publication. Retrieved June 4, 2010. doi: 10.3791/1791.

Cullins MJ, Shaw KM, Gill JP, Chiel HJ (2015) Motor neuronal activity varies least among individuals when it matters most for behavior. J Neurophysiol 113:981-1000.

Garcia S, Guarino D, Jaillet F, Jennings TR, Pröpper R, Rautenberg PL, Rodgers C, Sobolev A, Wachtler T, Yger P, Davison AP (2014) $\mathrm{Neo}$ : an object model for handling electrophysiology data in multiple formats. Front Neuroinformatics 8:10.

Gill JP, Chiel HJ (2020) Rapid adaptation to changing mechanical load by ordered recruitment of identified motor neurons. eNeuro. Advance online publication. Retrieved April 22, 2020. doi: 10. 1523/ENEURO.0016-20.2020.

Hurwitz I, Neustadter DM, Morton DW, Chiel HJ, Susswein AJ (1996) Activity patterns of the B31/B32 pattern initiators innervating the I2 muscle of the buccal mass during normal feeding movements in Aplysia californica. J Neurophysiol 75:1309-1326.

Islam MN, Martin SK, Aggleton JP, O'Mara SM (2019) NeuroChaT: a toolbox to analyse the dynamics of neuronal encoding in freely-behaving rodents in vivo. Wellcome Open Res 4:196.

Jacobson TK, Ho JW, Kent BW, Yang F-C, Burwell RD (2014) Automated visual cognitive tasks for recording neural activity using a floor projection maze. J Vis Exo. Advance online publication. Retrieved Feb 24, 2014. doi: 10.3791/51316.

Kluyver T, Ragan-Kelley B, Pérez F, Granger BE, Bussonnier M, Frederic J, Kelley K, Hamrick JB, Grout J, Corlay S, Ivanov P, Avila D, Abdalla S, Willing C (2016) Jupyter notebooks - a publishing format for reproducible computational workflows. In: Positioning and power in academic publishing: players, agents and agendas. Amsterdam: IOS Press.

Lu H, McManus JM, Chiel HJ (2013) Extracellularly identifying motor neurons for a muscle motor pool in Aplysia californica. J Vis Exp. Advance online publication. Retrieved Mar 25, 2013. doi: 10.3791/ 50189.

Lu H, McManus JM, Cullins MJ, Chiel HJ (2015) Preparing the periphery for a subsequent behavior: motor neuronal activity during biting generates little force but prepares a retractor muscle to generate larger forces during swallowing in Aplysia. J Neurosci 35:5051-5066.

McKinney W (2010) Data structures for statistical computing in Python. Proceedings of the 9th Python in Science Conference, pp 51-56. Available at http://conference.scipy.org/proceedings/ scipy2010/mckinney.html.

McManus JM, Lu H, Cullins MJ, Chiel HJ (2014) Differential activation of an identified motor neuron and neuromodulation provide Aplysia's retractor muscle an additional function. J Neurophysiol 112:778-791.

Morton DW, Chiel HJ (1993) In vivo buccal nerve activity that distinguishes ingestion from rejection can be used to predict behavioral transitions in Aplysia. J Comp Physiol A Neuroethol Sens Neural Behav Physiol 172:17-32.

van der Walt S, Colbert SC, Varoquaux G (2011) The NumPy array: a structure for efficient numerical computation. Comput Sci Eng 13:22-30.

Virtanen P, Gommers R, Oliphant TE, Haberland M, Reddy T, Cournapeau D, Burovski E, Peterson P, Weckesser W, Bright J, van der Walt SJ, Brett M, Wilson J, Millman KJ, Mayorov N, Nelson ARJ, Jones E, Kern R, Larson E, Carey CJ, et al. (2020) SciPy 1.0: fundamental algorithms for scientific computing in Python. Nat Methods 17:261-272. 